\title{
ON THE MICROWAVE SPECTRUM OF \\ METHANE IN THE ATMOSPHERES OF THE OUTER PLANETS
}

\author{
KENNETH FOX \\ Earth Resources and Astrophysics Laboratory, Dept. of Physics and Astronomy, \\ University of Tennessee, Knoxville, Tenn., U.S.A.
}

\begin{abstract}
The existence of a small dipole moment in the vibronic ground state of methane leads to transitions in the microwave spectral range. Frequencies and line strengths appropriate to the atmospheres of the outer planets are presented.
\end{abstract}

\section{Introduction}

Methane is known to be an important constituent of the atmospheres of Jupiter, Saturn (and its satellite Titan), Uranus, and Neptune (McElroy, 1969; Fox, 1972a; Newburn and Gulkis, 1973). Spectra of $\mathrm{CH}_{4}$ in the infrared and visible regions have been studied for a long time, and have yielded information on the characteristics of these atmospheres (Fox, 1972a). The optical spectra of $\mathrm{CH}_{4}$ usually arise from vibration-rotation transitions in the ground vibrational and electronic ('vibronic') state. In these transitions, the change of electric dipole moment ' $\mu$ ' with vibration permits absorption (or possibly emission) of electromagnetic radiation (Herzberg, 1945).

A tetrahedral (' $\left.T_{d}{ }^{\prime}\right)$ molecule like $\mathrm{CH}_{4}$ was conventionally assumed (Herzberg, 1945) to have no permanent $\mu$ in its ground vibronic state, by virtue of the high symmetry in this state. Consequently, $\mathrm{CH}_{4}$ was not expected to have pure rotational transitions, i.e., transitions in which only its rotational quantum numbers change. Spectra of $\mathrm{CH}_{4}$ in the far-infrared and microwave regions were thus thought to be forbidden.

Difference vibration-rotation spectra of $\mathrm{CH}_{4}$ may occur in the far-infrared (see Herzberg, 1945), but are expected to be relatively weak because of the Boltzmann factor.

Collision-induced far-infrared absorption spectra of $\mathrm{CH}_{4}$ have been discussed extensively by Ozier and Fox (1970); this paper contains references to earlier experimental and theoretical work. Such spectra depend on molecular collisions to permit absorption, and their strengths are not great at low gas pressures. However, collisioninduced absorption by mixtures of $\mathrm{CH}_{4}$ and $\mathrm{H}_{2}$ has recently been considered as a source of thermal opacity in the atmospheres of Jupiter, Saturn, Uranus, and Neptune (Fox and Ozier, 1971) and Titan (Pollack, 1973).

Pure rotational transitions in an excited vibrational state of $\mathrm{CH}_{4}$ were predicted by Mizushima and Venkateswarlu (1953) and one such transition was recently observed directly by Curl and Oka (1973); the latter paper contains references to earlier experimental and theoretical work. These excited-state transitions are also expected to be relatively weak because of the Boltzmann factor, as in difference spectra.

However, in 1971, it was theoretically predicted that pure rotational transitions in the vibronic ground state of $T_{d}$ molecules could occur as the result of a small $\mu$ arising 
from vibration-rotation interactions (Fox, 1971) or, alternatively, from centrifugal distortion effects (Watson, 1971; Aliev, 1971). This $\mu$ was measured (Ozier, 1971), by molecular-beam techniques, to have the magnitude $(5.38 \pm 0.10) \times 10^{-6} \mathrm{D}$ for ${ }^{12} \mathrm{CH}_{4}$. Subsequently, a far-infrared absorption spectrum corresponding to pure rotational $\Delta J=+1$ transitions (here $J$ represents the quantum number for the total angular momentum $\mathbf{J}$ ), arising from $\mu$ in the ground vibronic state of ${ }^{12} \mathrm{CH}_{4}$ was also reported (Rosenberg et al., 1972). More recently, a pure rotational $\Delta J=0$ transition of ${ }^{12} \mathrm{CH}_{4}$, produced by the same mechanism (Fox, 1971; Watson, 1971; Aliev, 1971) was observed by infrared-radio frequency double resonance (Curl et al., 1973).

In 1972, it was noted (Fox, 1972b), that in the absence of an $\mathbf{N H}_{3}$ atmospheric consituent on Saturn, Uranus, or Neptune, $\mathrm{CH}_{4}$ may be promoted as a source of microwave opacity there. This remark would apply to Titan also. A microwave spectrum was calculated and tabulated with absolute intensities corresponding to $T=0^{\circ} \mathrm{C}$ by Fox (1972b). Some similar results for $T=300 \mathrm{~K}$ were presented by Dorney and Watson (1972). As experimental evidence for pure rotational transitions in methane has increased, and because more refined spectroscopic constants needed to calculate spectral line positions with microwave accuracy are now available (Curl, 1973), it seems appropriate and useful to discuss in more detail, with calculations tailored to the atmospheres of the outer planets, microwave spectra of methane in those environments.

\section{Theory}

The strength of an individual absorption line is given by Fox (1972b)

$$
\alpha_{\mathrm{abs}}=\left(8 \pi^{3} / 3 h c\right)(N / \mathscr{Z})\left(E_{f}-E_{i}\right)<\mu_{J K}>{ }^{2} \varepsilon_{J K}\left(e^{-E_{i} h c / k T}-e^{-E_{f} h c / k T}\right),
$$

where $h$ and $k$ are Planck's and Boltzmann's constants, respectively, and $c$ is the speed of light in vacuum; $N$ is the number of molecules per $\mathrm{cm}^{3}$, and $\varepsilon_{J K}$ is the nuclear-spin statistical weight factor for the initial state of the transition. The dipole moment for $\Delta J=0$ transitions in the ground vibronic state is

$$
\begin{aligned}
\left\langle\mu_{J K}\right\rangle^{2} & =(3 / 2) C_{34}^{2}(2 J-2)(2 J-1)(2 J+1)(2 J+3) \times \\
& \times(2 J+4)[f(J K)]^{2},
\end{aligned}
$$

where $f(J K)$ is a linear combination of vector-coupling coefficients; $K$ is the projection of $\mathbf{J}$ (total angular momentum) on the molecule-fixed $z$-axis. The coefficient $C_{34}$ depends only on molecular parameters, in addition to universal constants.

The partition function $\mathscr{Z}$ may be approximated well for $T \leqslant 300 \mathrm{~K}$ by the rotational partition function $\mathscr{Z}_{r}$ which in turn may be expressed (Fox, 1970) as

$$
\mathscr{Z}_{r}=\left(4 \pi^{1 / 2} / 3\right)\left(B_{0} h c / k T\right)^{-3 / 2} e^{B_{0} h c / k T},
$$

a form accurate to $1 \%$ for $T>40 \mathrm{~K}$, for $\mathrm{CH}_{4} ; B_{0}$ is the ground-state rotational constant. The energies $E_{i}$ and $E_{f}$ refer to the initial and final states, respectively. The 
difference of Boltzmann factors in Equation (1) may be rewritten as

$$
e^{-E_{i} h c / k T}-e^{-E_{f} h c / k T} \equiv e^{-E_{i} h c / k T}\left[1-e^{-\left(E_{f}-E_{i}\right) h c / k T}\right] .
$$

A useful approximation to Equation (4) may be obtained as follows:

$$
\left(E_{f}-E_{i}\right) h c / k T=4.8 \times 10^{-5}\left(E_{f}-E_{i}\right) / T,
$$

where $E_{f}$ and $E_{i}$ are in units of $\mathrm{MHz}$, and $T$ is in $\mathrm{K}$. For $J \leqslant 20$, the range of the tabulation by Fox (1972b), the transition energies $E_{f}-E_{i}<130000 \mathrm{MHz}$. Consequently, for $T>45 \mathrm{~K}$, a possible effective temperature for Neptune (Newburn and Gulkis, 1973),

$$
\left(E_{f}-E_{i}\right) h c / k T<0.14 \text {. }
$$

Equation (4) may then be replaced, to an accuracy of at least $7 \%$, by

$$
e^{-E_{i} h c / k T}-e^{-E_{f} h c / k T}=e^{-E_{i} h c / k T}\left(E_{f}-E_{i}\right) h c / k T .
$$

As the absorption coefficient $\alpha_{\text {abs }}$ has already been tabulated by Fox (1972b) at $T=0^{\circ} \mathrm{C}$, for the present purposes it is essentially necessary only to recalculate its temperature-dependent part $\alpha_{\text {abs }}(T)$. From Equations (1), (3), and (7),

$$
\alpha_{\text {abs }}(T)=\mathscr{Z}_{r}^{-1} e^{-E_{i} h c / k T} h c / k T \text {. }
$$

The initial-state energy is given in the usual form (Herzberg, 1945),

$$
E_{i}=B_{0} J(J+1)-D_{s} J^{2}(J+1)^{2},
$$

where $D_{s}$ is the centrifugal distortion constant appropriate (Hecht, 1960) to $T_{d}$ molecules. Higher-order contributions (Hecht, 1960; Moret-Bailly, 1961) have been neglected in Equation (9). These include the $T_{d}$ fine-structure terms which determine the microwave transition energies, but which are characteristically (Moret-Bailly et al., $1965)$ much less than $1 \%$ of Equation (9) for methane.

Then from Equations (3), (8), and (9)

$$
\begin{gathered}
\frac{\alpha_{\mathrm{abs}}(T)}{\alpha_{\mathrm{abs}}(273.15)}=\left(\frac{273.15}{T}\right)^{5 / 2} \exp \left\{-\left[B_{0}\left(J+\frac{1}{2}\right)^{2}-D_{s} J^{2}(J+1)^{2}\right] \times\right. \\
\left.\times(h c / k T)\left(1-\frac{T}{273.15}\right)\right\} .
\end{gathered}
$$

This is the factor by which the absolute intensities in (Fox, 1972b) are to be multiplied to make them correspond to temperatures appropriate for the atmospheres of the outer planets.

Spectral line positions may be readily determined from the ground-state $T_{d}$ finestructure terms expressed (Moret-Bailly, 1965), to fourth order, as

$$
\begin{aligned}
E_{(J, p)}^{0}= & {[(2 J-3) \cdots(2 J+5)]^{1 / 2}\left[\varepsilon^{0}+\varrho^{0} J(J+1)\right](-1)^{J} F_{A_{1} p p}^{(4 J J)}+} \\
& +[(2 J-5) \cdots(2 J+7)]^{1 / 2} \xi^{0}(-1)^{J} F_{A_{1} p p}^{(6 J J)},
\end{aligned}
$$


where $\varepsilon^{0}, \varrho^{0}$, and $\xi^{0}$ are spectroscopic constants for the vibronic ground state. The $F$ functions are vector-coupling coefficients adapted to cubic symmetry. These functions have been tabulated numerically (Moret-Bailly et al., 1965) for $J \leqslant 21$. The subscripts $p$ label the $T_{d}$ sub-levels in a $J$ multiplet. Each transition frequency may be obtained from an energy difference of the form $E_{(J, p)}^{0}-E_{\left(J, p^{\prime}\right)}^{0}$.

\section{Results}

Of the many microwave transitions tabulated previously (Fox, 1972b), only those which fall in the frequency range observed for the outer planets (Newburn and Gulkis, 1973) are considered in the present study. Moreover, only the stronger transitions with $\alpha_{\mathrm{abs}}>10^{-12} \mathrm{~cm}^{-2}$ amagat $^{-1}$ at the relevant atmospheric temperatures are given here.

\section{TABLE I}

Spectral line positions and absolute line intensities for microwave absorption by methane in the atmospheres of the outer planets, for a characteristic temperature of $134 \mathrm{~K}$

Transition $^{a}$

$$
\begin{aligned}
6 & \rightarrow \leftarrow 6 \\
A 2 & \rightarrow \leftarrow A 1 \\
7 & \rightarrow \leftarrow 7 \\
F 1(1) & \rightarrow \leftarrow F 2(2) \\
8 & \rightarrow \leftarrow 8 \\
F 1(1) & \rightarrow \leftarrow F 2(2) \\
F 1(1) & \rightarrow \leftarrow F 2(1) \\
9 & \rightarrow \leftarrow 9 \\
A 2 & \rightarrow \leftarrow A 1 \\
F 1(1) & \rightarrow \leftarrow F 2(2) \\
F 2(2) & \rightarrow \leftarrow F 1(2) \\
10 & \rightarrow \leftarrow 10 \\
A 2 & \rightarrow \leftarrow A 1 \\
F 2(1) & \rightarrow \leftarrow F 1(1) \\
F 2(2) & \rightarrow \leftarrow F 1(2) \\
11 & \rightarrow \leftarrow 11 \\
E(1) & \rightarrow \leftarrow E(2) \\
F 1(2) & \rightarrow \leftarrow F 2(3) \\
F 1(1) & \rightarrow \leftarrow F 2(2) \\
12 & \rightarrow \leftarrow 12 \\
A 1(1) & \rightarrow \leftarrow A 2 \\
F 1(1) & \rightarrow \leftarrow F 2(2) \\
F 1(2) & \rightarrow \leftarrow F 2(3) \\
14 & \rightarrow \leftarrow 14 \\
E(1) & \rightarrow \leftarrow E(3) \\
E(1) & \rightarrow \leftarrow E(2) \\
15 & \rightarrow \leftarrow 15 \\
A 1(1) & \rightarrow \leftarrow A 2 \\
& \leftarrow \leftarrow-F 2
\end{aligned}
$$

Absolute intensity
$\left(\mathrm{cm}^{-2}\right.$ amagat

760.921

1562.879

2774.606

1563.809

1257.944

2546.742

1791.730

5014.758

3946.940

2688.583

4599.229

5169.506

7468.747

13276.228

10797.232

6940.206

27137.337

19281.566

21287.410

$$
1.51(-12)
$$$$
1.62(-12)
$$

$5.07(-12)$

$1.06(-12)$

$2.33(-12)$

$1.52(-12)$

$1.31(-12)$

4.44(-12)

$1.68(-12)$

$1.50(-12)$

$3.14(-12)$

$2.77(-12)$

$2.38(-12)$

2.83(-11)

$3.80(-12)$

$1.62(-12)$

$2.55(-12)$

$1.30(-12)$

$1.17(-12)$

a For convenience in tabulation, $F 2(1)$ denotes $F_{2}^{(1)}$, etc. (see Table II of Fox, 1972b).

b Powers of 10 are indicated in parentheses, e.g., $1.51(-\cdots 12) \equiv 1.51 \times 10^{-12}$. 
These restrictions may be relaxed as necessitated by future observations and/or models of the atmospheres of the outer planets and their satellites. Also, the atmospheres may be characterized by many different values of temperature, so that it is difficult to choose a particular one or even a small set; and it would be prohibitive to present the results for all possible temperatures here. Consequently, one probable characteristic temperature $T=134 \mathrm{~K}$ has been selected. The frequencies given in Table I are likely to include most of the stronger transitions for Jupiter, Saturn, Uranus, Neptune, and Titan. Modifications of the absolute intensities in Table I may readily be made by the application of Equation (10). With $T=134 \mathrm{~K}$, and the following values of groundstate rotational constants obtained in the analysis of a high-resolution vibrationrotation infrared spectrum (Barnes et al., 1972): $B_{0}=5.24059 \mathrm{~cm}^{-1}$ and $D_{s}=1.0855 \times$ $\times 10^{-4} \mathrm{~cm}^{-1}$; Equation (10) becomes

$$
\begin{aligned}
\alpha_{\mathrm{abs}}(134) / \alpha_{\mathrm{abs}}(273.15)= & (5.933) \times \\
& \times \exp \left[-0.0287(J+1 / 2)^{2}+5.94 \times 10^{-7} J^{2}(J+1)^{2}\right] .
\end{aligned}
$$

The squared magnitude of the dipole moment is taken (Ozier, 1971) to be $C_{34}^{2}=$ $=\left(5.38 \times 10^{-6} D\right)^{2}$, as in Table II of Fox $(1972 b)$.

Microwave transition frequencies had been obtained previously (Fox, 1972b) on the basis of a single (Hecht, 1960) ground-state spectroscopic constant $D_{t} \equiv-3.05505$ $\varepsilon^{0}$. A mean value of $D_{t}=4.403 \times 10^{-6} \mathrm{~cm}^{-1}$ was adopted on the basis of three determinations made directly from experimental data (Barnes et al., 1972; Husson and Dang Nhu, 1971; Ozier et al., 1970). More recently, however, a refined value of $\varepsilon^{0}$, together with accurate values of $\varrho^{0}$ and $\zeta^{0}$, have been deduced (Curl, 1973) from infrared-radio frequency double resonance observations of two $\Delta J=0$ transitions in the vibronic ground state of ${ }^{12} \mathrm{CH}_{4}$; the molecular-beam measurements of Ozier, (1971) were used in the analysis of Curl (1973). The values employed in Equation (11) of the present work are $\varepsilon^{0}=-43516.819_{1} \mathrm{~Hz}, \varrho^{0}=5.514_{1} \mathrm{~Hz}$, and $\xi^{0}=-0.452_{9} \mathrm{~Hz}$. These are only slightly different from Curl (1973). The spectral line positions in Table I have been determined from energy differences based on Equation (11) with the current values of $\varepsilon^{0}, \varrho^{0}$, and $\xi^{0}$ shown above. These tabulated frequencies may be expected to be accurate to at least $0.1 \mathrm{MHz} \equiv 10^{5} \mathrm{~Hz}$. The notation for $T_{d}$ sub-levels in MoretBailly (1965) and Moret-Bailly et al. (1965) differs from that in Table II (see footnote a) of Fox (1972b). The conventions used in the latter are followed here in Table I.

\section{Acknowledgements}

I am grateful to R. F. Cuil, Jr. for preprints and useful discussions of his papers. Partial support from the Fowler-Marion Fund for travel to the Extraordinary General Assembly of the International Astronomical Union in Poland (1973) is deeply appreciated.

Note added in proof. Recently some new work has appeared which is related to the present paper. Takami, M., Uehara, K., and Shimoda, K. (1973, Japan J. Appl. Phys. 
12, 924) observed two pure rotational transitions in the same excited vibrational state of $\mathrm{CH}_{4}$ utilized by Curl and Oka (1973). An explicit generalization of MizushimaVenkateswarlu (1953) transitions to excited states of all infrared-active transitions in $T_{d}$ molecules was given by Fox, K. (1974, J. Chem. Phys. 60, 337). The coincidence of Er:YAG laser emission with methane absorption was considered by Fox, K. (1974, Appl. Phys. Letters 24, 24) from the viewpoint of experiments and calculations related to the polar character of the ground and excited vibrational states. Holt, C. W., Gerry, M. C. L., and Ozier, I. (1973, Phys. Rev. Letters 31, 1033) observed four $\Delta J=0$ transitions in the ground vibronic state of methane in a microwave absorption spectrum. None of these measured lines corresponds to the transitions in Table I. However, it may be inferred that the predicted frequency for $E(2) \rightarrow \leftarrow E(3)$ of $J=14$ would differ from the measured value by a few $\mathrm{MHz}$, so that the frequencies given in Table I may be expected to be accurate to only several $\mathrm{MHz}$. The sixth-order analogue of Equation (11) has been developed by Michelot, F., Moret-Bailly, J., and Fox, K. (1974, J. Chem. Phys., to be published). Tarrago, G., Dang Nhu, M., and Poussigue, G. (1974, Compt. Rend. Acad. Sci. Paris 278B, 207) have determined values of $\varepsilon^{0}, \varrho^{0}$, and $\xi^{0}$ which differ somewhat from those used in the present work, and which may lead to transition frequencies varying from those in Table I by several $\mathrm{MHz}$. The parameters $B_{0}$ and $D_{s}$ in Equations (3), (9), and (10) have been re-evaluated by Tarrago, G., Dang Nhu, M., and Poussigue, G. (1974, J. Mol. Spectrosc. 49, 322) from new high-resolution infrared spectra; however, their changes are not expected to have any effect on the absolute intensities in Table I to the stated accuracy.

\section{References}

Aliev, M. R.: 1971, Zh. Eksperim. Teor. Fiz. Pis'ma Red. 14, 600.

Barnes, W. L., Susskind, J., Hunt, R. H., and Plyler, E. K.: 1972, J. Chem. Phys. 56, 5160.

Curl, R. F., Jr. and Oka, T.: 1973, J. Chem. Phys. 58, 4908.

Curl, R. F., Jr., Oka, T., and Smith, D. S.: 1973, J. Mol. Spectrosc. 46, 518.

Curl, R. F., Jr.: 1973, J. Mol. Spectrosc. 48, 165.

Dorney, A. J. and Watson, J. K. G.: 1972, J. Mol. Spectrosc. 42, 135.

Fox, K.: 1970, J. Quant. Spectrosc. Radiat. Transfer 10, 1335.

Fox, K.: 1971, Phys. Rev. Letters 27, 233.

Fox, K. and Ozier, I.: 1971, Astrophys. J. 166, L95.

Fox, K.: 1972a, in K. N. Rao and C. W. Mathews (eds.), Molecular Spectroscopy: Modern Research, Academic Press, New York, p. 79.

Fox, K.: 1972b, Phys. Rev. A6, 907.

Hecht, K. T.: 1960, J. Mol. Spectrosc. 5, 355, 390.

Herzberg, G.: 1945, Infrared and Raman Spectra of Polyatomic Molecules, Van Nostrand, Princeton, N.J.

Husson, N. and Dang Nhu, M.: 1971, J. Phys. (Paris) 32, 627.

McElroy, M. B.: 1969, J. Atmospheric Sci. 26, 798.

Mizushima, M. and Venkateswarlu, P.: 1953, J. Chem. Phys. 21, 705.

Moret-Bailly, J.: 1961, Cahiers Phys. 15, 237.

Moret-Bailly, J.: 1965, J. Mol. Spectrosc. 15, 344.

Moret-Bailly, J., Gautier, L., and Montagutelli, J.: 1965, J. Mol. Spectrosc. 15, 355.

Newburn, R. L., Jr. and Gulkis, S.: 1973, Space Sci. Rev. 3, 179.

Ozier, I. and Fox, K.: 1970, J. Chem. Phys. 52, 1416.

Ozier, I., Yi, P. N., Khosla, A., and Ramsey, N. F.: 1970, Phys. Rev. Letters 24, 642. 
Ozier, I.: 1971, Phys. Rev. Letters 27, 1329.

Pollack, J. B.: 1973, Icarus 19, 43.

Rosenberg, A., Ozier, I., and Kudian, A. K.: 1972, J. Chem. Phys. 57, 568.

Watson, J. K. G.: 1971, J. Mol. Spectrosc. 40, 536.

\section{DISCUSSION}

Irvine: Can any of the microwave lines be measured with existing instrumentation?

Fox: Definitely yes in the laboratory (see references in my paper). For atmospheres, I don't know yet. The answer will depend on atmospheric conditions such as the abundance of methane and masking by other molecules. 